\title{
Ovarian, Fallopian Tube, and Primary Peritoneal Carcinoma TNM Finding v8
}

National Cancer Institute

\section{Source}

National Cancer Institute. Ovarian, Fallopian Tube, and Primary Peritoneal Carcinoma

TNM Finding v8. NCI Thesaurus. Code C139927.

A finding about one or more characteristics of ovarian, fallopian tube, or primary peritoneal carcinoma, following the rules of the TNM AJCC v8 classification system. Nonepithelial primary ovarian cancers may be staged using this classification but should be reported separately. (from AJCC 8th Ed.) 